\title{
Les enjeux identitaires dans la trilogie de crise de Linda Lê
}

\section{Claude Cavallero et Université Savoie Mont Blanc}

\section{(2) OpenEdition}

10 Journals

\section{Édition électronique}

URL : https://journals.openedition.org/studifrancesi/22089

DOI : 10.4000/studifrancesi.22089

ISSN : 2421-5856

Éditeur

Rosenberg \& Sellier

\section{Édition imprimée}

Date de publication : 1 avril 2020

Pagination : $67-75$

ISSN : 0039-2944

\section{Référence électronique}

Claude Cavallero et Université Savoie Mont Blanc, « Les enjeux identitaires dans la trilogie de crise de Linda Lê », Studi Francesi [En ligne], 190 (LXIV | I) | 2020, mis en ligne le 01 avril 2021, consulté le 03 août 2021. URL : http://journals.openedition.org/studifrancesi/22089; DOI : https://doi.org/10.4000/ studifrancesi.22089

\section{(c) (i) $\odot$}

Studi Francesi è distribuita con Licenza Creative Commons Attribuzione - Non commerciale - Non opere derivate 4.0 Internazionale. 


\title{
Les enjeux identitaires dans la trilogie de crise de Linda Lê
}

\begin{abstract}
Linda Lê's novelistic works - shaped by several conflicting influences - reveal a problematic cultural identity, and her conflicting points of view nourish a rebellious writing style, as dazzling in its metaphorical and dreamlike fluidity as is the daze it can inspire. This article deals with the ambiguities deriving from the quest for identity unfolding through the texts inspired by the suffering born from the exile that led the author to declare in an interview: «I have the impression of carrying in me a dead body. It is surely Vietnam that I carry as a dead child». This outstanding piece of work is a testament to the psychological difficulties upon which writings of migrant authors often reflect. Focusing on the trilogy devoted to the death of her father, composed of the three novels: Les Trois Parques; Voix, une crise; Lettre morte, the article aims to enlighten the path of writing through which real resilience is achieved by the novelist, in order to finally be able to mourn, to overcome the dilemma of not being able to return to the native country, by opening up to new ways of finding her identity instead of having to make amends with the painful past and trying to be reborn under the auspice of a conciliating tone.
\end{abstract}

À bien des égards, l'œuvre de Linda Lê apparaît déconcertante. En quête d'une identité insaisissable, de nombreuses pages de ses romans confrontent le lecteur à une virtuosité stylistique époustouflante, laquelle ne peut que susciter la perplexité. C'est à juste titre que la critique a souligné le baroquisme de son écriture ainsi que la complexité de son inspiration où les références classiques et mythologiques se mêlent aux allusions tacites à la culture vietnamienne $e^{1}$. La lecture de Linda Lê requiert parfois cette qualité d'attention que Thomas Pavel compare à une forme de dévotion dont seuls seraient capables «certains lecteurs qui aiment l'insolite et apprécient les défis culturels $»^{2}$. Une telle patience sera de mise pour venir à bout des trois ouvrages que l'auteure consacre à la disparition de son père resté au Vietnam, et qui marquent une période charnière dans sa vie d'exilée comme dans son parcours de création. Ces textes ont pour titres Les Trois Parques, Voix: une crise, et Lettre morte $e^{3}$. Publiés respectivement en 1997, 1998 et 1999, ils retracent les étapes douloureuses d'un deuil dont Lê a cru ne jamais pouvoir se remettre. Nous nous proposons d'analyser ici les enjeux identitaires qui sous-tendent l'écriture de ce triptyque à vocation quasi thérapeutique. À l'instar d'un miroir grossissant, le prisme de la mort associée au fantôme paternel, le spectre menaçant de la folie et la tentation du suicide y confèrent une acuité singulière aux questions que pose l'œuvre entière de l'écrivaine s'agissant

(1) Voir la thèse de Thi Thu Thuy Bui, La crise de l'exil chez Linda Lê, thèse de doctorat en Lettres et Arts, Université Lumière Lyon 2, 2012, p. 291.

(2) T. Pavel, La Pensée du roman, Paris, Gallimard, 2003, p. 396.

(3) L. Lê, Les Trois Parques, Paris, Christian Bourgois, 1997; Voix: une crise, Paris, Christian Bourgois, 1998; Lettre morte, Paris, Christian Bourgois, 1999. Dans la suite de la présente étude seront utilisées les abréviations $T P$, $V x$, et $L M$. 
du rapport à la langue française, ce fameux complexe de Caliban qui ne cesse de la hanter ${ }^{4}$, ainsi que des modalités fictionnelles par lesquelles s'exprime l'ambivalence liée aux souffrances de l'exil.

Quelle que soit sa motivation propre, le lecteur ne saurait rester indifférent aux tensions multiples qui animent cette écriture du doute et de l'imprécation, en particulier dans Les Trois Parques, premier volume de la trilogie qui nous intéresse. Ce récit polyphonique dépourvu de mention générique (mais dont on perçoit clairement le substrat autobiographique) met en scène deux sœurs et leur cousine exilées en France tandis que le père des sœurs mène une vie misérable à Saïgon. Les jeunes femmes s'apprêtent à recevoir cet homme abandonné par sa famille dans la «rutilante» maison de l'aînée des sœurs installée en région parisienne. Un certain dimanche après-midi, toutes trois s'affairent dans la cuisine pour préparer des recettes vietnamiennes. Cet huis-clos d'allure théâtrale induit un véritable imbroglio de pulsions émotionnelles et de flash-backs ouvrant la brèche d'une nostalgie restée inavouable et encore tenue à distance par le jeu de l'ironie. De page en page, la fluidité métaphorique des impressions ressenties nous surprend, de même que les ruptures des registres de langue associant les termes argotiques et les expressions populaires à l'hypercorrection de tournures savantes. L'élan des digressions suggèrent l'emballement, la perte de contrôle d'une énergie rebelle face à l'impossible acceptation d'une mutilation dont la jeune cousine, surnommée «la Manchote» incarne le symbole physiques. D'une manière incongrue, le père lui-même fait l'objet de railleries sous la plume d'une narratrice grimacière qui n'hésite pas à superposer son point de vue à celui des protagonistes, comme on le voit à travers ces lignes évoquant la cousine et la sœur cadette, appelée «belles gambettes» en raison de ses longues jambes:

(...La Manchote n'avait pas bougé de sa place près de l'évier. Les bras croisés, elle fixait le ventre rond, penché au-dessus du congélateur, tout occupé à compter les encornets en hibernation et les blocs de carne givrée. Quelle plaie, le dimanche, entre la montgolfière et le bois mort!) S'il n'y avait pas eu les vacances du roi Lear à l'ordre du jour, les longues jambes se seraient taillées depuis belle lurette. Mais pas moyen d'échapper à l'ennui. Ni au vieillard décharné, qu'il allait falloir soustraire à sa maison de poupée, cueillir à sa descente d'avion et trimbaler dans les rues de la ville (TP, p. 20).

Les motifs domestiques qui prolifèrent au fil des lignes placent le récit sous le signe d'une oralité discursive qui n'est pas sans rappeler la phrase délirante de Céline, non plus que les improvisations digressives de Beckett ou de Valère Novarina. Une telle dextérité rhétorique témoigne de surcroît de la grande maitrise de la langue d'adoption dans laquelle Linde Lê a fait le choix déterminé de s'exprimer dans ses livres. Cerner la portée identitaire de ce choix nécessite de prendre en compte l'enfance vietnamienne de Linda Lê en rappelant sa découverte précoce de la langue française dans une institution au nom délicieux de «Couvent des Oiseaux». L'auteure décrit, dans Le Complexe de Caliban, l'exaltation profonde que lui procura cet apprentissage, sans rien cacher du revers de médaille que suppose un tel apanage:

(4) Linda Lê a consacré un ouvrage au «complexe de Caliban», d'après La Tempête de Shakespeare, où elle compare la situation de l'écrivain ayant choisi d'écrire dans une langue autre que sa langue maternelle à celle d'un esclave que la plus totale soumission à son maître ne parvient jamais à défaire d'intentions hérétiques. L. Lê, Le Complexe de Caliban, Paris, Christian Bourgois, 2005, p. 102. Dans la suite de la présente étude sera utilisée l'abréviation CDC.

(5) Thi Thu Thuy Bui souligne la fonction narrative d'intensification dramatique du «moignon» de ce personnage dont le moi s'exprime dans la malformation ou le manque. Cf. T. Bui, La Crise de l'exil chez Linda Lê cit., p. 199. 
Le fait que, vivant au Vietnam, je parlais et lisais le français, me condamnait à une sorte d'exil intérieur. J'avais à la fois la certitude et la hantise de ma différence. De vouloir écrire dans une langue que ne maîtrisait pas mon père équivalait à un reniement de mes origines. Ce sentiment de désaveu, je n'en avais qu'une perception obscure. Mais ce fut l'exaltation qui l'emporta quand les bouleversements politiques m'obligèrent, à quatorze ans, à me séparer de mon père et à quitter le pays pour rejoindre la France $(C D C$, p. 11$)$.

La langue française, explicitement associée au culte des livres, ouvre à l'enfant les portes d'un imaginaire émancipateur dont elle ne pourra plus se détacher. D'où la douleur qu'éprouvera l'adolescente contrainte de sacrifier sous la pression de «l'agent de purification communiste» sa collection de Oui-Oui, ses contes d'Andersen et son volume des Misérables. «On m'avait volé, dit-elle, la plus belle part de mon enfance» $(C D C$, p. 15). Au moment de se séparer de son père et de quitter le Vietnam pour gagner la France, l'attirance de Linda Lê se dénue d'ambiguiité: «Je dois avouer que la douleur d'être arrachée à mon père parlait moins fort que l'excitation éprouvée à débarquer dans ce pays découvert à travers les livres» (CDC, p. 11).

Cet aveu explique, bien des années plus tard, le rejet symbolique du Vietnam que l'on peut constater à la lecture des livres de Linda Lê. Un pays dont le nom apparaît pour la première fois seulement dans Les Trois Parques (texte qui constitue son cinquième ouvrage). Or, ce refus implicite d'aborder frontalement la question de l'exil ne signifie pas, on le devine, que le thème du déracinement, comme impossible oubli, puisse être expurgé de l'écriture. Certes l'écriture permet-elle à l'auteure de se forger une identité culturelle dans laquelle la littérature figure l'espace emblématique d'une patrie, ainsi que Linda Lê le confie dans un entretien avec Catherine Argand'. Toutefois, l'invocation d'une telle patrie «extra-territoriale» ne saurait masquer la rémanence, en filigrane du texte et comme à la manière d'une fantomatique voix «off», de multiples traces de la langue et de la culture vietnamiennes. Dans sa thèse déjà citée, Thi Thu Thuy Bui mentionne les influences de la langue vietnamienne sur la désignation périphrastique des personnages, sur l'usage de la synonymie en forme de jeu langagier, de même que la référence culturelle aux rites funéraires (le blanc couleur du deuil) ainsi qu'à des croyances ancrées dans l'imaginaire (les fantômes et les sorcières) ${ }^{7}$. Il est donc évident que le vietnamien n'est pas pour Linda Lê une langue totalement oubliée, comme elle a pu le déclarer parfois, et que le Vietnam en tant que sémiosphère n'est pas davantage ce «corps mort» double d'elle-même qu'elle évoque dans l'entretien déjà cité avec Catherine Argand ${ }^{8}$.

Par-delà les modalités fictionnelles qui caractérisent ce texte à forte connotation biographique, une certaine "vietnamitude" se fait jour dans Les Trois Parques

(6) Linda Lê, entretien avec Catherine Argand, "Lire" 148, avril 1999, pp. 36-37. Cette assimilation symbolique de la langue d'écriture au pays d'accueil, et donc à la sphère littéraire comme seul refuge existentiel possible pour l'écrivain(e) a maintes fois été invoqué, par Cioran (que cite Linda Lê dans CDC, p. 91), mais aussi notamment par J.M.G. Le Clézio et A. Djebar. Cf. J.M.G. Le Clézio, Éloge de la langue française, "L'Express" 2205, 7-13 oct. 1993, pp. 82-83; A. Djebar, Ces voix qui m’assiègent..., Paris, Albin Michel, 1999, p. 215.

(7) Comme cela est souvent le cas en Asie, la motivation sémantique des patronymes est fréquente en vietnamien. Thi Thu Thuy Bui indique que le prénom même que s'est choisi l'auteure, Linda, signifie en vietnamien «beaucoup d'âme» (Linh Đa), ce qui ne peut que référer à son expérience interculturelle... Elle évoque aussi la coutume consistant à brûler de faux billets de banque à chaque anniversaire de la mort d'un défunt, afin qu'il ne manque de rien dans l'au-delà. T. Bui, La crise de l'exil chez Linda Lê cit., pp. 63-66, 180-184.

(8) Dans Le Complexe de Caliban, Linda Lê évoque son choix linguistique au cours d'un bref chapitre qui se termine par une conclusion on ne peut plus claire: «J'avais tué le mandarin mais son fantôme hantait mon encrier». $C D C$, p. 53. 
au gré d'observations critiques de la société vietnamienne contemporaine, concernant notamment les enjeux sociaux révélés par le prisme des rapports familiaux. Ces renvois à la réalité du pays d'origine sont introduits dans la diégèse par le biais des notations relatives à la nourriture, de sorte qu'un lien subreptice s'établit entre habitus culinaire et espace mémoriel. La satisfaction des besoins alimentaires, faut-il le préciser, est longtemps restée une préoccupation importante au Vietnam en raison de la famine ayant causé deux millions de morts pendant la seconde guerre mondiale, et également à cause de la malnutrition liée à la présence militaire américaine dans le Sud jusqu'en 1975. Dans sa maison tombée en décrépitude, le père, alias le roi Lear, souffre visiblement du manque de nourriture, même s'il préfère cultiver des fleurs plutôt que des légumes dans son jardin et réclame pour cela des bulbes de tulipes à l'aînée de ses filles:

Ça l'avait pris brusquement. Une lubie. Il y avait bien dix ans. À l'époque, les saprophytes ne poussaient pas encore à Sägon. Tout autour de la maison bleue, les vieux palais étaient encore debout, mais la ville crevait de faim. Le roi Lear aussi. La faim lui était sûrement montée au cerveau, dit l'ainée de mes cousines. Il n'avait qu'une idée fixe, la culture des fleurs. Au lieu de réclamer des colis de survie, le roi Lear s'était fait envoyer des bulbes de tulipes (TP, p. 18).

La figure du père, cela n'étonne guère, est porteuse du lien identificatoire au pays d'origine et à l'enfance heureuse. Cependant ce père, dans le premier volume de la trilogie, concentre des polarités affectives contradictoires et globalement dysphoriques, étant le plus souvent moqué et dévalorisé à travers les mots d'humeur - et d'humour - des trois jeunes femmes. À l'évidence, la réconciliation de l'ailleurs et de l'origine perdue demeure à ce stade un leurre, une véritable chimère. Par le registre émotionnel de l'exaltation, voire de la colère, s'énonce l'ineffable difficulté de la quête identitaire. Aux dernières pages des Trois Parques, l'expression de la mort du père concrétise sur le ton de la fantaisie une impossible résilience: à l'instant de mourir, celui-ci n'est plus que «le roi Lear» ou «l'acteur au costard» (LTP, p. 240). La mort elle-même est d'ailleurs désignée sur un mode onirique, sans que le vocable ne soit jamais utilisé:

Le roi Lear était parti à l'aube sur sa bécane qui faisait du surplace. L'orage avait éclaté dans la nuit et une averse chaude cinglait les fleurs de l'enclos, qui piquaient du nez, s'abattaient face contre terre. La pluie s'infiltrait par le toit, gouttait sur la table, faisant une flaque au milieu de l'anthologie ouverte depuis des jours à la même page [...]. Le roi Lear, sur son engin diabolique, fila rapide comme l'éclair qui traçait dans le ciel des guirlandes de feu (TP, p. 240).

En guise du voyage de retrouvailles avec ses filles, voyage dont l'organisation logistique s'imposait jusque-là comme prétexte essentiel du récit, le départ définitif du père referme le premier volet de cette trilogie selon une tonalité dénégatoire qui scelle l'étape première d'un parcours psychique associé au deuil. Cette mort symbolique entérine la mise à distance énonciative qu'opère au fil du texte la désignation mythologique des personnages (les Parques) et l'emprunt à Shakespeare de la figure symbolique du «roi Lear»?.

Sous le titre Voix, une crise, le second volet de la trilogie annihile cette distance pour nous plonger brutalement dans l'épisode dépressif qui fait suite à l'impossible

(9) À noter qu'à l'instar des filles de Lear (Regan, Gonerill et Cordelia) dans la tragédie de Shakespeare, les deux sœurs des Trois Parques sont orphelines de mère. C'est cependant l'aînée, et non la cadette, qui s’identifie à Cordelia dans l'œuvre de Linda Lê. 
acceptation de la mort du père. Le récit, assumé par une narratrice homodiégétique, s'ouvre sur une scène confuse ayant pour cadre hypothétique un centre de soins psychiatriques. «Je ne sais pas où je suis, note-t-elle. Dans un centre de crise, comme on m'a dit, ou dans un théâtre avec des comédiens qui jouent leur partie et m'enrôlent en me laissant le choix des répliques» $(V x, \mathrm{p} .7)$. Telle une panoplie de masques interchangeables, des femmes défilent le long d'un corridor où seule résonne l'incohérence de leurs propos. La séquence «Je ne sais pas où je suis» impose peu à peu son leitmotiv, associant le lecteur à une quête éperdue du sens - comme dans les pièces de Beckett où cette recherche est sans cesse contrariée... L'anonyme narratrice partage la chambre d'une prétendue philosophe convaincue que «la Création est défectueuse» et «l'amour impossible» ( $V x$, p. 10); au travers d'un soupirail à barreaux, elle ne perçoit du monde extérieur que les jambes de passants empressés dont il est impossible de suivre l'itinéraire:

De mon lit face au soupirail, j'entends le vacarme des allants et venants, je vois un monde sans tête, le flot agité des piétons qui vont de l'avant pendant que je voyage immobile dans mon lit comme au fond d'une barque qui sombre, avec ma tête qui s'emballe, s'égare, perd le nord, résonne de fragments de phrases. Vous avez le bac? Sidonie a plus d'un amant, mon bébé, mon chéri, On est en prison ici, si Dieu me rend visite je Lui cracherai à la gueule ( $V x$, p. 14).

Cette désorientation nous conduit malgré tout à l'appartement de la narratrice, autre lieu clos où le fantôme du père défunt lui rend visite d'une manière insistante qui devient vite obsessionnelle. Lorsqu'elle s'abandonne à la dérive dans les rues d'un Paris vaguement mythique - on songe à Nadja - elle doit lutter pour déjouer les pièges d'une secrète «Organisation». Ici et là surgissent des voix, «voix épouvantables et que personne n'entend» $(V x$, p. 25), lesquelles finiront par l'obliger à brûler les lettres de son père ( $V x$, p. 26). Plusieurs scènes de cauchemar s'enchaînent, mêlant les images morbides aux gestes de persécution, exhortant parfois même à l'automutilation. Comme l'on pouvait s'y attendre, la perte des repères entraîne la jeune femme sur les pentes vertigineuses de la folie.

Il devient évident que le vide laissé par la disparition du père exacerbe les enjeux identitaires restés en suspens dans le premier volet de la trilogie. Ce vide révèle un sentiment de culpabilité qui se manifeste en particulier lors de certaines hallucinations focalisées sur la maison familiale, comme dans les lignes suivantes: «Je suis au pays de mon enfance. Je cherche la maison aux volets bleus. Il ne reste plus qu'un tas de cendres. Des lettres brillent au fond, voyelles mutilées, consonnes aux jambages arrachés. Je plonge ma main, remue la cendre, d'où monte une voix, Tu l'as tué» $(V x$, p. 47).

Du fait qu'elle n'était pas au chevet de son père au moment de son agonie, la narratrice se sent responsable d'un acte de trahison: ne pas avoir pris l'avion pour lui rendre une ultime visite, c'est l'avoir clairement abandonné ${ }^{10}$. Alors le remords la ronge, la plonge dans une spirale d'autodestruction. Elle se replie sur elle-même puis s'abandonne au délire. Les images les plus funestes s'enchaînent comme les scènes d'un film d'horreur - cadavres d'oiseaux morts, têtes coupées jetées à la Seine, chiens venus de l'Enfer pour se délecter des mares de sang $(V x$, p. 35, 41)... Ce foyer spéculatif d'épouvante motive le rapport énonciatif de l'écriture à la folie, illustrant l'intérêt

(10) Dans le troisième volet de la trilogie, Lettre morte, ce sentiment d'abandon sera explicité et référé au départ en exil. Cf. $L M$, p. 54. 
non dissimulé de Linda Lê pour des écrivains rongés par l'angoisse de la démence (Hölderlin, Byron, Nerval, Artaud....) ${ }^{11}$.

Au seuil extrême de la folie, l'écriture devient le lieu d'une errance multiple, liée à la fois à la géographie et à l'espace du souvenir. L'errance spatiale dans les rues de la capitale se conjugue aux déambulations imaginaires dans la ville vietnamienne, comme si nul refuge, nulle certitude existentielle ne pouvait endiguer la peur panique qui étreint "l'héroïne" à chaque minute. Ainsi confie-t-elle:

J'erre dans les rues du pays de mon enfance. La ville est déserte. Partout ruines et cendres. Mon père apparaît, disparaît entre les ruines. Je suis sa trace. Je pénètre dans un zoo. Les cages sont vides. Je longe un pont. Le fleuve est à sec. Les arbres des jardins, calcinés, tendent leurs bras noirs vers le ciel. Dans les rues, les hurlements des chiens à trois têtes me poursuivent. Le bruit de moteur fait mal à mes oreilles. L’Organisation a saccagé la ville, pour que je ne puisse trouver refuge nulle part ( $V x$, p. 48).

Au plus fort de la crise, un nouveau refrain scande les visions hallucinatoires, le pressentiment qu'une balle perdue de revolver viendra fatalement se loger dans son crâne pour mettre une fin définitive au supplice ${ }^{12}$. S'en remettre à un tel aléa est le signe désespéré qui entérine du point de vue fictionnel une situation limite dont il est aisé d'admettre le caractère emblématique: la double exclusion de l'exilée se donne à lire dans l'impossibilité de trouver la sérénité dans le pays où elle vit non plus que dans celui qu'elle a quitté. D'où la tentation de se mettre en quête d'un nouvel ailleurs hypothétique, plusieurs fois exprimée au fil des pages - «Je dois partir», «Je dois repartir» $(V x, \mathrm{p} .52,53,69)^{13}$. Mais l'on voit qu'il ne s'agit là que d'un simple réflexe de défense psychique mettant à jour une absence de but: «Je suis comme une enfant, conclura in fine la narratrice, qui a peur et ne sait pas où aller» $(V x$, p. 65).

Nous touchons ici une problématique essentielle que pose toute situation d'exil, et que nous pouvons assimiler à une impossibilité foncière d'habiter le pays d'accueil, tandis que le territoire familier du pays natal est devenu hors de portée. Dans ses Réflexions sur l'exil, Edouard W. Said se réfère au témoignage d'Adorno pour analyser ce détachement contraint de l'exilé dont la résolution ne pourrait être qu'illusoire, le philosophe affirmant avec une grave ironie: «Il fait aussi partie de la morale de ne pas habiter chez soi» ${ }^{14}$. Aucune issue concrète ne peut dès lors s'offrir à la narratrice. Malgré elle, la voici condamnée à l'existence bornée de ce métèque que Linda Lê décrit habilement dans Le Complexe de Caliban, lequel, «quand il se regarde dans la glace, ne voit qu'un moi falsifié, une identité niée, des manières d'enfant naturel en quête de légitimité» $(C D C$, p. 31). Cet être-métèque, sous les traits hypostasiés d'une grenouille rejouant le dédoublement du Dr Jekyll et de Mr Hyde, abrite le démon du voyageur sans famille, parasite malade de «la souffrance de n'être ni d'ici ni d'ailleurs» et du sentiment «d'avoir usurpé sa place» $(C D C$, p. 33). D’une façon

(11) Linda Lê s'est maintes fois exprimée à ce propos dans ses entretiens, et s'agissant de son propre itinéraire, elle va jusqu'à noter, dans Le Complexe de Caliban: «L'histoire de mon âme est celle de l'exil, du deuil et de la folie qui les accompagne» (CDC, p. 86).

(12) On trouve la première occurrence d'une telle pensée obsessionnelle vers la fin du second chapitre: «J'attends la balle qui viendra me frapper dans le dos ou en pleine tête» (Vx, p. 23).

(13) L'attirance d'un autre ailleurs virtuel n'est jamais très loin chez Linda Lê. Dans l'entretien déjà cité avec Catherine Argand, elle déclare: «Je suis sur le qui-vive, je veux savoir toujours vivre en exil. En ce moment, je rêve de partir ailleurs, pour redoubler l'exil, ne plus vivre dans le pays dont je pratique la langue». Linda Lê, entretien avec C. Argand cit., p. 37.

(14) E.W. Said, Réflexions sur l'exil et autres essais [2000], Actes Sud, 2008, p. 255. «Les réflexions d'Adorno, note à ce propos Said, sont empreintes de la conviction que l'écriture est aujourd'hui la seule manière accessible, bien que fragile et vulnérable, d'être chez soi» (ibidem). 
comparable, la crise engendrée par le deuil du père accentue le mal-être et lui confère une tournure singulière. Il deviendra en l'occurrence impossible à la jeune femme de prendre l'avion pour retourner au pays d'enfance. À l'image d'un acte manqué, elle se met à errer de long en large dans l'aéroport de Roissy, puis, ne sachant plus où aller, elle finit par rentrer chez elle ${ }^{15}$.

Le choc de la disparition du père a ébranlé les fragiles certitudes de l'être. Il a éveillé le dilemme profond de l'exil par lequel, entre perte et découverte, entre attirance et nostalgie, l'individu se révèle non identique à lui-même. Un espace «d'entredeux» s'insinue, pour reprendre la notion féconde du psychanalyste Daniel Sibony selon qui «toutes nos situations cruciales sont sous-tendues par une situation d'entredeux, posture instable ou incrustée dont l'épreuve semble décisive» ${ }^{16}$. Après le déni et la crise s'ouvre dans Lettre morte la possibilité d'un nécessaire apaisement, d'une articulation devenue indispensable entre des fragments d'identités multiples. Tant que le rappel de l'origine exacerbé par la mort du père était trop brûlant et traumatique pour être vécu en tant que tel, l'écriture ne pouvait être qu'explosive, sarcastique (Les Trois Parques) ou maintenue sous pression hallucinatoire (Voix, une crise). Remarquons que Lettre morte est le seul texte de la trilogie adressé à un narrataire explicite, dénommé Sirius - il s'agit d'un ami confident à qui la narratrice raconte son histoire au cours d'une soirée interminable. Le récit se déploie en un long fil monologué, un discours linéaire que rien ne vient interrompre, comme issu d'un seul souffle, offrant par là un saisissant contraste avec l'éclatement énonciatif des visions de Voix, une crise comme avec l'emmêlement des points de vue observable dans Les Trois Parques. À l'instar d'une oraison funèbre, la parole qui se libère entre culpabilité et nostalgie ne répugne pas à désigner la mort: absents des premiers volumes, le mot «mort» et le verbe «mourir» font irruption dès les premières pages du livre. Cette acceptation littérale de la perte du père laisse dès lors augurer d'un possible rétablissement.

En quoi l'invocation constitue-t-elle précisément une «lettre morte»? Voici un point sur lequel l'auteure cultive l'ambiguïté. Nous savons qu'une missive du père s'était perdue dans Les Trois Parques, qu'une secrète organisation avait ordonné la destruction de toutes les lettres dans Voix, une crise. La narratrice de Lettre morte s'interroge incidemment: «à quoi bon ce monologue impromptu, cette lettre que je lui écris pour remplacer toutes celles que je n'ai pas écrites de son vivant?» ( $L M$, p. 18). On ne sait par ailleurs que penser de cette missive que le père tente lui-même d'écrire avant de mourir:

Sur son lit d'hôpital, mon père essaya de m'écrire une dernière lettre. Elle ne m'est jamais parvenue. Il avait demandé du papier, avait esquissé quelques mots, puis avait laissé tomber le crayon. Que cherchait-il à me dire? Cette lettre que je n'ai pas reçue, j'y réponds maintenant en te parlant. Mais l'entend-il? Mes mots de remords lui parviennent-ils? L'apaisent-ils? Non, je le sens qui s'agite, exige de moi que je déballe mes secrets, fouille mes plaies, plonge le couteau dans mon cœur, vide mes entrailles ( $L M$, p. 47).

Peut-être l'instance titulaire de cet opus de clôture forme-t-elle tout simplement un métonyme de l'œuvre en cours, œuvre du détachement désormais privée de son prime destinataire, ainsi que le laisse entendre Linda Lê en réponse à une question de Marie-Françoise Colombani: «les véritables lettres à mon père, ce sont mes livres» ${ }^{17}$,

(15) Ce détail institue des limites à la porosité biographique du texte, puisque pour sa part Linda Lê est retournée pour la première fois au Vietnam lors du décès de son père, en 1995.

(16) D. Sibony, Entre-deux, l'origine en partage, Paris, Seuil, 1991, p. 15.

(17) M-F. Colombani, Linda Lê: l'âme à vif, "Elle" 7 septembre 1998, p. 52. 
propos en outre repris et développé dans l'entretien avec Catherine Argand ${ }^{18}$. Quoi qu'il en soit, cette lettre qui n'en est pas une ne cesse pour autant d'être voyageuse au sens du mouvement d'aller-retour entretenu entre les souvenirs de l'enfance heureuse au Vietnam, sous la protection tutélaire du père, et la vie difficile à Paris où la narratrice endure une relation amoureuse clandestine et houleuse avec un personnage au nom aussi ironique qu'éloquent, puisqu'il s'appelle Morgue! À la manière de plans parallèles au cinéma se jouent les scènes d'un drame dont l'héroïne est la victime toute désignée. D'un côté, la douleur du deuil s'amplifie du regret d'avoir abandonné le père:

Il est mort seul, il a vécu seul. Sa solitude m'accuse. J'aurais pu me rendre au pays de mon enfance, m'approcher de la maison où mon père m'attendait. Mais je suis restée de ce côtéci de l'océan. Je l'ai laissé mourir seul sans l'avoir jamais revu. Le jour où mon père mourut, le téléphone sonna ici très tôt le matin, et une voix me lut un télégramme. La formule, en vietnamien, disait non la mort, mais la perte ( $L M$, p. 16).

D'autre part, la frustration de vivre une passion sans issue où son intégrité de femme se trouve bafouée la réduit à un état de dépendance et la condamne à une solitude paradoxale qui l'incite à commettre l'irréparable. Page après page, la souffrance oscille ainsi entre remords et répulsion, désespoir et révolte. Ce mouvement de balancier constitue la toile de fond sur laquelle se poursuit textuellement la quête identitaire de la narratrice, dont nous comprenons qu'elle ne saurait déboucher sur aucune véritable catharsis. Car le pays de l'origine ne peut offrir une solution de repli synonyme d'identité pleine et durable. Ainsi que l'énonce Linda Lê à propos du lord Jim de Conrad, dans Tu écriras sur le bonbeur, «revenir chez soi, c'est un peu "venir rendre des comptes". Mais un écrivain ne rend jamais de comptes. Il trahit la terre, la langue, le nom du père» ${ }^{19}$. En conséquence, le seul apaisement possible pour la protagoniste (double de l'auteure) passe par l'amputation d'une partie d'elle-même, c'est-à-dire de son passé, idée clairement exprimée dans la clausule résolutive:

Je suis en train de mourir d'un mot qui n'a pas été dit. Ce mot, est-ce le mot d'amour que Morgue a fait miroiter et que mon père a murmuré, attendant en vain que je le répète? Dans l'obscurité qui est désormais la mienne, $\mathrm{j}$ 'avance en tâtonnant. Des fantômes dansent autour de moi. L'amour m'a quittée, la mort chante une cantilène lugubre. Je suis en deuil de moi-même, nostalgique de cette enfance que mon père a emportée dans sa tombe (LM, p. 104).

La notion de «tâtonnement» apparaît ici très importante. Elle signifie que l'espace dynamique d'entre-deux institué par l'écriture permet le déplacement/dépassement autrement qu'en fixant la problématique de l'origine sur une réponse définitive. L'image du personnage de Bartleby, le jeune scribe dissident de Melville auquel Linda Lê se réfère dans Le Complexe de Caliban, et en lequel elle se découvre «une sorte de frère» $(C D C$, p. 45$)$, s'avère parfaitement éloquente: ce n'est pas la certitude établie, l'acquiescement péremptoire ou le refus rigide qui peuvent guider vers la lumière le geste d'écriture, mais plutôt l'émergence d'un questionnement que suggère l'indécision rebelle du fameux «I would prefer not to $»^{20}$. Refermant la trilogie de crise, la

(18) «Tant que mon père était en vie, tous mes livres lui étaient adressés. Il était mon lecteur idéal, mon lecteur imaginaire. Lorsqu'il est mort, j'ai perdu mon lecteur, le ciel est devenu désert, le monde sans Dieu». Linda Lê, entretien avec Catherine Argand cit., p. 36.

(19) L. Lê, Tu écriras sur le bonbeur [1999], Paris, Christian Bourgois, 2009, p. 61.

(20) H. Melville, Bartleby, une histoire de Wall Street [1856], traduction de J. Vidal, Paris, Éditions Amsterdam, 2007. 
confession de Lettre morte s'achève aux premières lueurs d'un nouveau jour, symbole explicite d'une seconde naissance pour la narratrice ( $L M$, p. 105). Rien n'est encore inscrit sur la trame de ce jour neuf où tout peut arriver, mais la flamme résiliente du partage identitaire y transparaît déjà en filigrane.

CLAUDE CAVALLERO

Université Savoie Mont Blanc 